\title{
Rare Association of Eosinophilic Pancreatitis and Ulcerative Colitis: A Case Report and a Review of the Literature
}

\author{
Samlani $Z^{1}$, Lemfadli $\mathrm{Y}^{1^{*}}$, Bouchrit $\mathrm{S}^{1}$, Habiyaremye $\mathrm{C}^{1}$, Ait Errami $\mathrm{A}^{1}$, Oubaha $\mathrm{S}^{2}$, Rais $\mathrm{H}^{3}$, Krati $\mathrm{K}^{1}$
}

${ }^{1}$ Gastroenterology Department, Mohammed VI University Hospital, Marrakech

${ }^{2}$ Physiology Department, Faculty of Medicine and Pharmacy at Cadi Ayyad University, Marrakech

${ }^{3}$ Pathology Department, Mohammed VI University Hospital, Marrakech

DOI: $10.36347 /$ sjmcr.2020.v08i01.029

| Received: 20.01.2020 | Accepted: 27.01.2020 | Published: 30.01 .2020

*Corresponding author: Yassine Lemfadli

Abstract

Introduction: Eosinophilic pancreatitis is a rare cause of pancreatitis. It is characterized by localized or diffuse infiltration of the pancreatic gland by eosinophilic polymorphonuclear cells. It poses the problem of differential diagnosis with a pancreatic tumor, hence the interest of histological evidence by invasive means. Case report: We present a case of eosinophilic pancreatitis in a 37-year-old man who was diagnosed after an endoscopic biopsy in front of an obstructive mass of the head of the pancreas simulating a pancreatic tumor. In contrast to the data in the literature, our patient had only eosinophilic infiltration of the pancreas with no history of symptoms related to hypereosinophilia or to a high level of immunoglobulins E. The second peculiarity in our patient is the concomitant revelation of ulcerative colitis. Conclusion: Eosinophilic pancreatitis is a rare and often misdiagnosed clinical situation. Correct and precise diagnosis avoids unnecessary interventions. Echo-endoscopy with sample for histological study presents a fundamental means for the diagnosis of eosinophilic pancreatitis.

Keywords: Eosinophilic pancreatitis, eosinophils, ulcerative colitis, echo-endoscopy.

Copyright @ 2020: This is an open-access article distributed under the terms of the Creative Commons Attribution license which permits unrestricted use, distribution, and reproduction in any medium for non-commercial use (NonCommercial, or CC-BY-NC) provided the original author and source are credited.

\section{INTRODUCTION}

Eosinophilic pancreatitis (PE) is an extremely rare etiology of pancreatitis. It is characterized by diffuse or localized eosinophilic infiltration of the pancreas and high levels of IgE. It poses a real differential diagnostic problem with pancreatic cancer. Echo-endoscopy presents an important means for the diagnosis. We report a new case of eosinophilic pancreatitis associated with ulcerative colitis.

\section{Case Report}

We report the case of a 37-year-old man. He had no medical history. He was admitted for a permanent mucocutaneous jaundice with dark urine and discolored stools without pruritus evolving for 2 months. He also had intermittent rectal bleeding without other symptoms. He reported an unquantified weight loss and that he was apyretic. The clinical examination revealed a patient in good general condition with a WHO score of 1 and body mass index at $22 \mathrm{~kg} / \mathrm{m} 2$. He had mucocutaneous jaundice with no other abdominal exam abnormalities including no pain or palpable mass and no peripheral lymphadenopathy.

The biological assessment had objectified: a hemoglobin at $12.9 \mathrm{~g} / \mathrm{L}$; number of leukocytes at 5230/mm3; 3260 neutrophils (62.3\%); eosinophils: 230 (4.4\%) (reference range: 40 - 500); total bilirubin 19.9 mg / dL; alkaline phosphatase at $425 \mathrm{U} \mathrm{/} \mathrm{L;}$ gammaglutamyl transferase at $80 \mathrm{U} / \mathrm{L}$; ASAT at $225 \mathrm{U}$ / L; ALAT at $376 \mathrm{U} / \mathrm{L}$. Lipaemia was at 122 (<3 times normal). Hepatitis serology and serum IgG 4 assay were negative.

The abdominal ultrasound had shown a swollen pancreas in its cephalic portion with dilation of the intra- and extra hepatic bile ducts. The magnetic resonance cholangiography (BILI-MRI) had objectified a swollen aspect of the head of the pancreas which arrives in contact with the superior mesenteric vein without signal anomalies. It also showed significant dilation of the main bile duct and the intrahepatic bile ducts without vascular invasion and without dilation of the pancreatic ducts (figure1). 

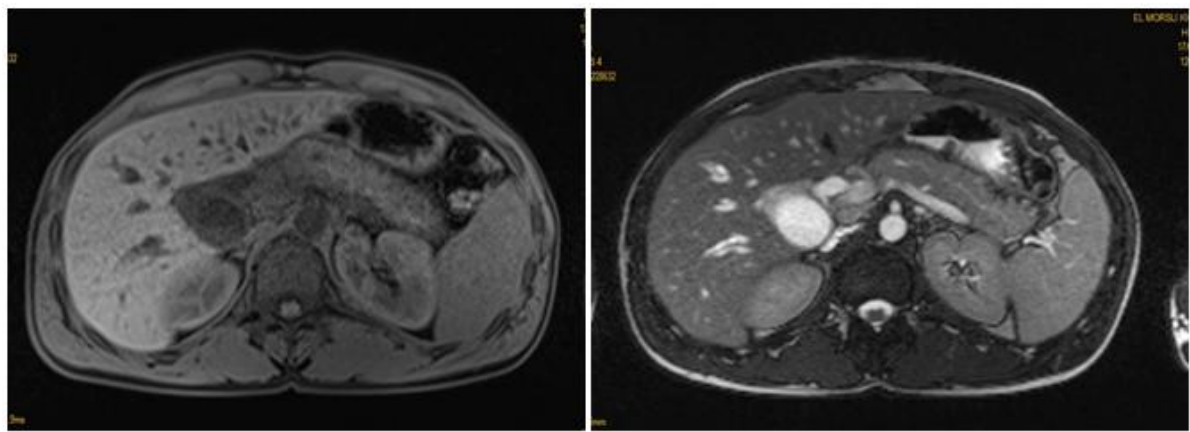

Fig-1: Bili-MRI: showing a swollen head of the pancreas with significant dilation of the main bile duct and the intrahepatic bile ducts

Endoscopic ultrasound examination showed a homogeneous hypoechoic tissue formation of the head of the pancreas measuring $3 \mathrm{~cm}$ without vascular invasion. She also showed dilation of the main bile duct and intrahepatic bile ducts and a dilated non-lithiasic thin-walled gallbladder (figure 2). A pancreatic mass cytopuncture was performed with a 22 Gauge needle. The histological study identified fibrosis of the pancreatic tissue with infiltration by eosinophils in an inflammatory polymorphic infiltrate (figure 3 ).

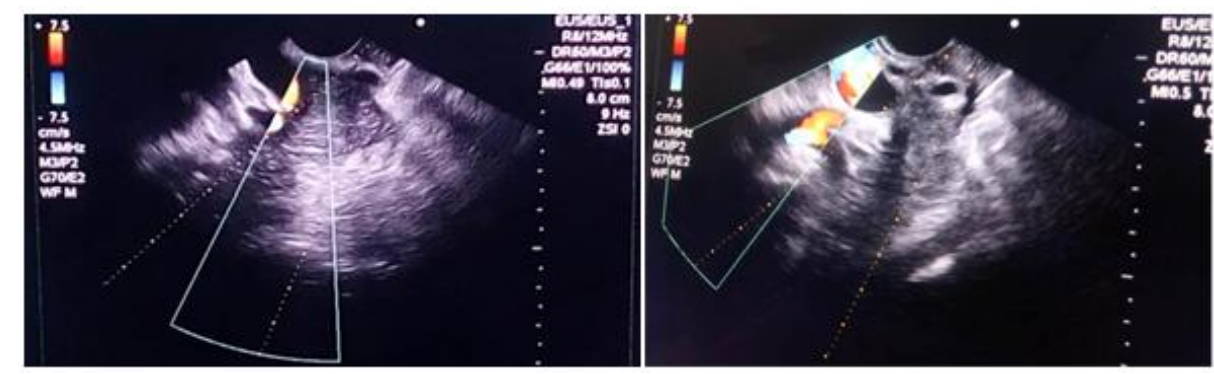

Fig-2: endoscopic ultrasound showing a hypoechoic mass of the head of the pancreas
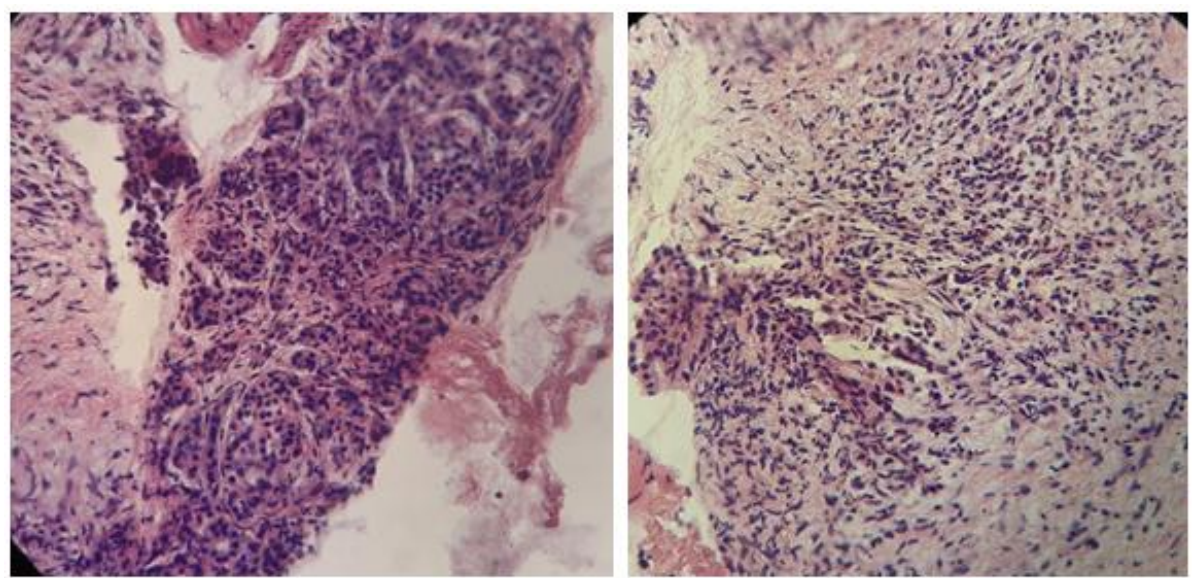

Fig-3: Pancreatic infiltration by eosinophils

Given the symptomatology and the imaging data, a tumor of the head of the pancreas was the most likely diagnosis. Ultrasound endoscopy with biopsy made it possible to rule out maliginity and to make the diagnosis of pancreatitis with eosinophils.

A colonoscopy done as part of the rectorragia had objectified a fragile rectal mucosa erythematous in one piece without interval of healthy mucosa from the anorectal line to the recto-sigmoid hinge. A sessile polypoid process was also found above the budding ileocecal valve, the site of ulceration at its apex (figure 4). Multiple biopsies were performed. Histological analysis of the process did not show signs of malignancy. Whereas rectal biopsies showed an ulcerated epithelium resting on a chorion which sits of an inflammatory infiltrate with basal plasmocytosis with highlighted lesions of cryptites and cryptic abscesses. 

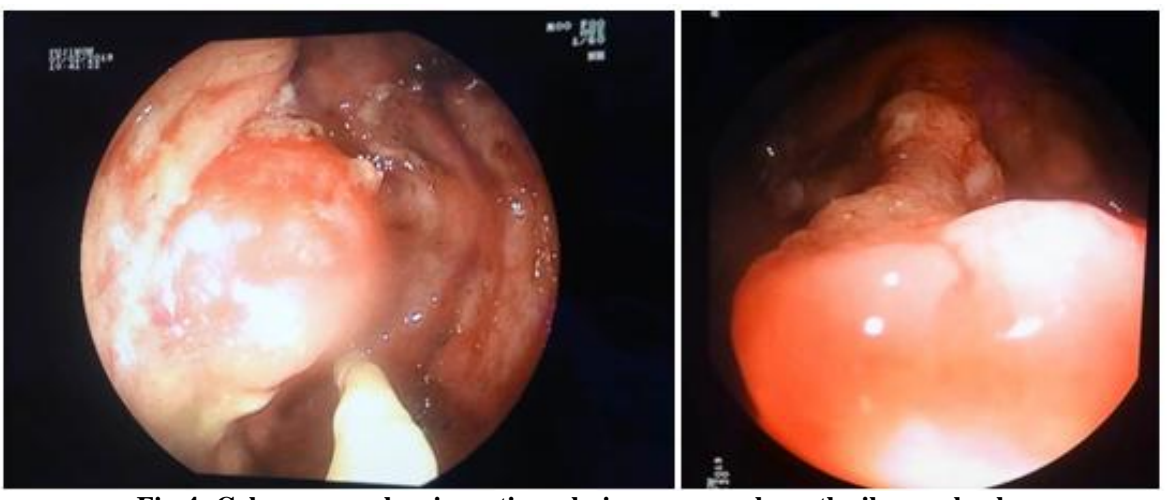

Fig-4: Colonoscopy showing a tissue lesion process above the ileocecal valve

The management consisted of a corticosteroid full dose oral with degression as an initial treatment and local mesalazine as a maintenance treatment for ulcerative colitis. The evolution was marked by the remission of jaundice and rectal bleeding. An abdominal control scan done two months later did not show any abnormalities in the pancreas (figure 5). A total colonoscopy of control showed a recto-colic mucosa without anomalies.
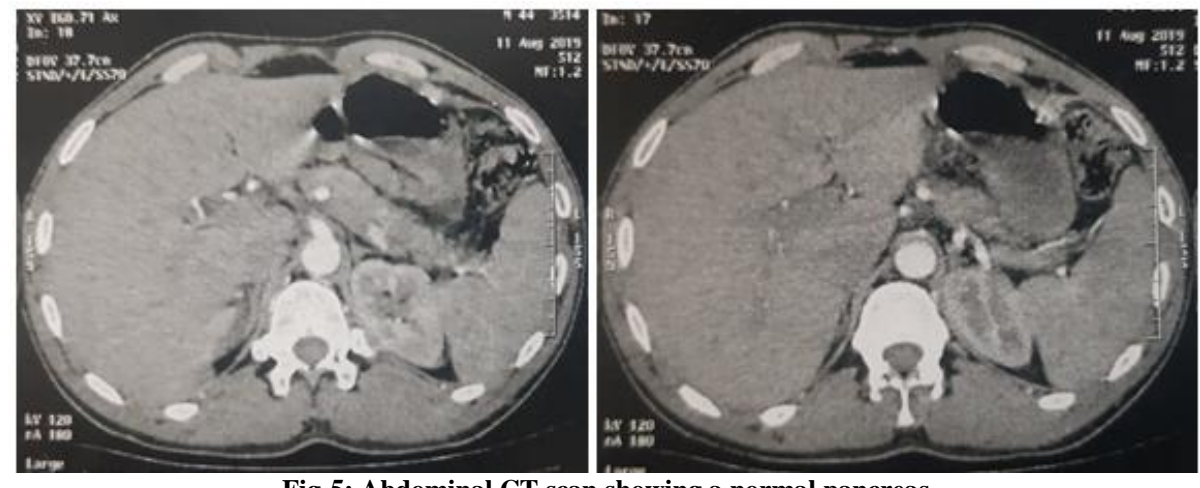

Fig-5: Abdominal CT scan showing a normal pancreas

\section{DISCUSSION}

$\mathrm{PE}$ is a rare etiology of chronic pancreatitis characterized by diffuse eosinophilic infiltration of the pancreas and increased serum IgE [1]. Usually, the patient presents for obstructive jaundice caused by a pancreatic mass due to inflammatory eosinophilic infiltration as in our case $[1,2]$. Many etiologies of PE have been suggested. Published cases include malignant neoplasms, parasitic infections, hypersensitivity to certain drugs (for example, carbamazepine), milk allergy, atopic terrain, newborn of a diabetic mother and association with hypereosinophilic syndrome or gastroenteritis to eosinophils [1,3].

The diagnosis of PE is often made on the basis of a hypereosinophilic syndrome or criteria of eosinophilic gastroenteritis in patients with gastrointestinal symptoms who present with eosinophilic infiltrations in histological / cytological samples [1, 4, 5].

The diagnostic criteria for hypereosinophilic syndrome are as follows: number of peripheral eosinophils greater than $1.5 \times 109$ for at least 6 months; medical history of rhinitis, asthma or other allergic diseases; eosinophilic infiltration of other organs (digestive system, skin and / or heart); excluding other causes of eosinophilia such as parasitic infection or leukemia $[1,5]$.

The main differential diagnosis arises with autoimmune pancreatitis (PAI) and chronic pancreatitis (CP). Eosinophilic pancreatitis is difficult to distinguish from PAI because they present clinical symptoms and similar radiological results $[1,6]$. Some cases of PC are associated with eosinophilia, which can make the diagnosis of one or the other difficult [1].

The final diagnosis depends on the histological and cytological results, with no radiological signs of CP. It is also based on the presence of a history of hypereosinophilic syndrome or eosinophilic gastroenteritis and the response to corticosteroids $[4,7]$.

Often PE is misdiagnosed as a malignant tumor and the diagnosis is only repaired after surgery. Echo-endoscopy with fine needle aspiration currently presents a good alternative and avoids excessive surgery $[8,9]$.

Medical treatment should be started as soon as the diagnosis is confirmed [10]. Steroids are the 
mainstay of PE medical therapy [11]. Oral prednisolone has been the cornerstone of treatment, with doses up to $40 \mathrm{mg} /$ day [4]. Improvement is usually noted within the first two to three weeks. Most patients do not have a recurrence [12]. Various other agents such as sodium cromoglycate (membrane stabilizing mast cell), ketotifen (antihistamine) and montelukast (a selective drug and leukotriene receptor antagonist) have been used with variable variables [12-14]. The installation of prosthesis or a sphincterotomy can be discussed in patients with biliary obstruction.

\section{Conclusion}

Eosinophilic pancreatitis is a rare and often misdiagnosed clinical situation. Its diagnosis is based on clinical, biological, radiological and histology examinations. Correct and precise diagnosis avoids unnecessary interventions. Echo-endoscopy with sample for histological study presents a fundamental means for the diagnosis of PE.

\section{REFERENCES}

1. Tian L, Fu P, Dong X, Qi J, Zhu H. Eosinophilic pancreatitis: Three case reports and literature review. Molecular and clinical oncology. 2016 Apr 1;4(4):559-62.

2. Barthet M, Hastier P, Buckley MJ, Bernard JP, Sastre B, Baroni JL, Salducci J, Delmont J. Eosinophilic pancreatitis mimicking pancreatic neoplasia: EUS and ERCP findings--is nonsurgical diagnosis possible? $\quad 1998$ Nov;17(4):419-22.

3. Barresi G, Inferrera C, De Luca F. Eosinophilic pancreatitis in the newborn infant of a diabetic mother. Virchows Arch A Pathol Anat Histol. 1978; 380: 341 - 348

4. Le Connie D, Nguyen H. Eosinophilic gastroenteritis, ascites, and pancreatitis: a case report and review of the literature. South Med J. 2004; 97: 905 - 906

5. Valent $\mathrm{P}$, Klion AD, Horny HP, Roufosse F, Gotlib J, Weller PF, Hellmann A, Metzgeroth G, Leiferman KM, Arock M, Butterfield JH. Contemporary consensus proposal on criteria and classification of eosinophilic disorders and related syndromes. Journal of Allergy and Clinical Immunology. 2012 Sep 1;130(3):607-12.

6. De Moura DT, de Paula Rocha RS, Jukemura J, Brunaldi VO, Guedes HG, Torrez FR, Ribeiro IB, Gelrud A, De Moura EG. A rare non-oncological pancreatic mass: eosinophilic pancreatitis diagnosis through EUS-FNA. Endoscopy international open. 2019 Feb;7(02):E151-4.

7. Gerritsen A, Molenaar IQ, Bollen TL, Nio CY, Dijkgraaf MG, van Santvoort HC, Offerhaus GJ, Brosens LA, Biermann K, Sieders E, de Jong KP. Preoperative characteristics of patients with presumed pancreatic cancer but ultimately benign disease: a multicenter series of 344 pancreatoduodenectomies. Annals of surgical oncology. 2014 Nov 1;21(12):3999-4006.

8. Kakodkar S, Omar H, Cabrera J, Chi K. Eosinophilic pancreatitis diagnosed with endoscopic ultrasound. ACG case reports journal. $2015 \mathrm{Jul} ; 2(4): 239$.

9. Brugge WR. Fine needle aspiration of pancreatic masses: the clinical impact. The American journal of gastroenterology. 2002 Nov 1;97(11):2701.

10. Sheikh RA, Prindiville TP, Pecha RE, Ruebner $\mathrm{BH}$ : Unusual presentations of eosinophilic gastroenteritis: case series and review of literature. World J. Gastroenterol. 2009; 15:2156-61.

11. Caglar E, Karismaz K, Dobrucali A: A case of eosinophilic gastroenteritis mimicking gastric lymphoma associated with pancreatitis due to duodenal involvement. Turk. J. Gastroenterol. 2012; 23:585-9.

12. Suzuki S, Homma T, Kurokawa M, Matsukura S, Adachi M, Wakabayashi K, Nozu F, Tazaki T, Kimura T, Matsuura T, Fukuda M. Eosinophilic gastroenteritis due to cow's milk allergy presenting with acute pancreatitis. International archives of allergy and immunology. 2012;158(Suppl. 1):7582.

13. Bastid C, Sahel J, Choux R, Payan MJ, Sarles H: Eosinophilic pancreatitis: report of a case. Pancreas. 1990; 5:104-7.

14. Roy-Peaud F, Paccalin M, Le Moal G, Landron C, Roblot P, Becq-Giraudon B: [Eosinophilic pancreatitis]. Presse Med. 2002; 31:25. 